03,08

\title{
Рассеяние электронов и дырок глубокими примесями в полупроводниковых гетероструктурах с квантовыми ямами
}

\author{
() Ю.А. Померанцев
}

Воронежский государственный педагогический университет, Воронеж, Россия

E-mail: pomerant_yu@mail.ru

Поступила в Редакцию 17 апреля 2020 г.

В окончательной редакции 17 апреля 2020 г.

Принята к публикации 2 июня 2020 г.

Исследовано рассеяние электронов и дырок системой глубоких примесей в полупроводниковых гетероструктурах на основе арсенида галлия с двумя квантовыми ямами в приближении предельно локализованного потенциала при произвольном профиле легирования. Показано, что зависимости вероятностей рассеяния в единицу времени для электронов и дырок от энергии носителя повторяют кусочно-постоянный вид плотности состояний подзон размерного квантования гетероструктуры с учетом вклада интеграла перекрытия волновых функций носителя. Для дырочных подзон с отрицательной эффективной массой вероятности рассеяния в единицу времени у краев подзон имеют сингулярности, характерные для одномерных систем.

Ключевые слова: полупроводниковые гетероструктуры, глубокие примеси, эффективная масса электрона, короткодействующий потенциал примеси.

DOI: 10.21883/FTT.2020.10.49903.092

\section{1. Введение}

Глубокими примесями определяются многие электронные, оптические и магнитные свойства полупроводников, поэтому проблеме глубоких примесных центров уделяется большое внимание [1-4]. Соединения GaNAs, GaInNAs и GaInNAsSb за последние два десятилетия привлекают большое внимание исследователей из-за их уникальных свойств и потенциала применения в современных оптоэлектронных устройствах, особенно в высокоэффективных многопереходных солнечных элементах, инфракрасных лазерах и фотоприемниках. Основная задача таких исследований - объяснение влияния глубокоуровневых дефектов на производительность и параметры будущих устройств $[5,6]$. Несмотря на это, продвижение в направлении получения количественных результатов, относящихся к конкретным примесям или иным точечным дефектам, например, вакансиям, пока незначительно. Моделирование точечных дефектов представляет чрезвычайно трудную задачу, и соответствующие результаты, как правило, не в состоянии дать сколько-нибудь полного и надежного описания поведения дефектов в полупроводниках.

С другой стороны, для полупроводниковой гетероструктуры (ГС) имеется физически наглядная картина формирования электронного энергетического спектра и квантовых состояний, обусловленных наличием примеси, в связи с особенностями зонной структуры полупроводника [7], основанная на последовательном учете вкладов различных энергетических зон в величину энергии связи электрона (или дырки) на примеси, что позволяет сформулировать полуфеноменологическое описание глубоких примесных состояний в рамках известного метода потенциала нулевого радиуса [8].

Целью настоящей работы является вычисление зависимостей вероятностей рассеяния в единицу времени (обратных времен релаксации) на глубокой примеси для электрона и дырки в нижних подзонах размерного квантования полупроводниковых ГС от энергии носителя в приближении предельно локализованного потенциала примеси с учетом межподзонного рассеяния носителей.

\section{2. Вероятность рассеяния электрона и дырки в единицу времени на глубоком примесном центре}

Рассмотрим переходы между электронными состояниями полупроводниковой ГС в 2D-подзонах размерного квантования, вызываемые наличием короткодействующего потенциала примесных атомов, который запишем как

$$
U(r)=-U_{0} a^{3} \sum_{l} q_{l} \delta\left(\boldsymbol{\rho}-\boldsymbol{\rho}_{l}\right) \delta\left(z-z_{0 l}\right) .
$$

Здесь: $U_{0}$ - глубина потенциальной ямы примесного центра, $a$ - постоянная решетки ГС, индекс $l$ пробегает по всем элементарным ячейкам кристалла, случайная функция $q_{l}$ равна единице, если в $l$-й ячейке находится примесный атом, и нулю - в противном случае, $z_{0 l}-$ координата примеси вдоль оси $z$ ГС, $\rho$ - поперечный (в плоскости $x y$ ) радиус-вектор носителя. 
Огибающие функции одноэлектронных волновых функций в ГС с квантовыми ямами (КЯ) могут быть записаны в виде [9]:

$$
|\lambda k\rangle=\frac{1}{\sqrt{S}} \exp (i k \rho) f_{\lambda k}(z),
$$

где $S$ - нормировочная площадь ГС; индекс $\lambda \equiv\{s, n\}$ определяет принадлежность к данной $s$-зоне объемных состояний и одновременно указывает номер $n$ подзоны размерного квантования для данной $s$-зоны; $k-$ поперечная составляющая квазиимпульса носителя. Функции $f_{\lambda k}(z)$ являются решением простого одномерного уравнения Шредингера, в котором потенциальная энергия моделируется соответствующим набором прямоугольных КЯ конечной глубины. Также предполагается, что на гетерограницах эти функции удовлетворяют граничным условиям, сохраняющим плотность потока вероятности, что и определяет энергетический спектр электронов и дырок ГС. Зависимостью 1D огибающей функции в (2) от 2D-квазиимпульса будем пренебрегать, ограничивая таким образом, рассмотрение случаем малой передачи квазиимпульса при рассеянии, $\Delta k \ll \pi / a$.

Вероятность перехода в единицу времени (обратное время релаксации) из состояния $|n k\rangle$ в состояние $\left|n^{\prime} k^{\prime}\right\rangle$ с помощью золотого правила Ферми можно записать в виде

$$
W_{n^{\prime} n}(k k)=\frac{2 \pi}{\hbar}\left|\left\langle n^{\prime} k^{\prime}|U| n k\right\rangle\right|^{2} \delta\left[E_{n^{\prime}}\left(k^{\prime}\right)-E_{n}(k)\right] .
$$

Здесь $E_{n}(k)$ есть энергетический спектр одноэлектронных возбуждений в ГС с КЯ; матричный элемент, входящий в (3), в принятом здесь приближении записывается как

$$
\left\langle n^{\prime} k^{\prime}|U| n k\right\rangle=\frac{U_{0} a^{3}}{S} \sum_{l} q_{l} f_{n^{\prime}}^{*}\left(z_{0 l}\right) f_{n}\left(z_{0 l}\right) \exp \left[i\left(k-k^{\prime}\right) \rho_{l}\right] .
$$

Вероятность в единицу времени перехода носителя из состояния $|n k\rangle$ в любое доступное состояние запишем в виде

$$
W_{n}(k)=\sum_{n^{\prime} k^{\prime}} W_{n^{\prime} k^{\prime}}\left(k^{\prime}, k\right),
$$

при этом предполагается, что концентрация примесей в $n^{\prime}$-подзоне достаточно мала, так что ограничениями, связанными с принципом Паули, можно пренебречь.

С учетом того, что уровень легирования ГС может быть достаточно высок (при селективном легировании, например, концентрация примесей в отдельных слоях ГС может быть величиной, близкой к единице), представляет интерес рассмотрение рассеяния не какимлибо конкретным пространственным распределением, а, скорее, некоторым усредненным распределением, учитывающим среди прочих факторов межпримесные корреляции. Следовательно, произведения $q_{l} q_{l^{\prime}}$ в уравнении (5) необходимо заменить на соответствующие средние значения: $q_{l} q_{l^{\prime}} \Rightarrow\left\langle q_{l} q_{l^{\prime}}\right\rangle$. В дальнейшем, мы ограничимся рассмотрением наиболее интересного случая однородно легированных слоев, которые перпендикулярны к оси роста ГС. Кроме того, для удобства, можно ввести вместо дискретного аргумента случайной функции $q_{l}$ непрерывные переменные: $q_{l} \Rightarrow q(\rho, z)$. Тогда функция $q(z)=\int d^{2} \rho q(\rho, z)$ будет представлять собой среднюю атомную концентрацию примесных атомов в слое между $z$ и $z+a$. Соответственно, флуктуация примесной концентрации в этом слое определится как $\delta q(\rho, z)=q(\rho, z)-q(z)$. Опуская случаи, которые соответствуют отсутствию рассеяния, мы можем переписать уравнение (5) в следующем виде:

$$
\begin{aligned}
W_{n}(k)= & \frac{2 \pi}{\hbar}\left(U_{0} a\right)^{2} \sum_{n^{\prime} k^{\prime}} \sum_{l l^{\prime}}\left\langle\delta q\left(k-k^{\prime}, z_{0 l}\right) \delta q^{*}\left(k-k^{\prime}, z_{0 l^{\prime}}\right)\right\rangle \\
& \times R_{n n^{\prime}}\left(z_{0 l}, z_{0 l^{\prime}}\right) \delta\left(E_{n^{\prime}}\left(k^{\prime}\right)-E_{n}(k)\right) .
\end{aligned}
$$

Здесь

$$
R_{n n^{\prime}}\left(z_{0 l}, z_{0 l^{\prime}}\right)=f_{n}^{*}\left(z_{0 l}\right) f_{n}\left(z_{0 l}\right) f_{n^{\prime}}\left(z_{0 l^{\prime}}\right) f_{n^{\prime}}^{*}\left(z_{0 l^{\prime}}\right),
$$

a 2D-фурье-компоненты примесных флуктуаций концентрации определяются выражением

$$
\delta q(k, z)=\frac{1}{S} \int d^{2} \rho q(\rho, z) \exp (i k \rho) .
$$

Корреляционная функция, содержащаяся в уравнении (6), может быть представлена в виде суммы, включающей вклады двух различных видов: вклада, который соответствует идеальному твердому раствору и другого вклада, связанного со всеми корреляционными эффектами, как в слоях, так и вдоль оси роста ГС. Учитывая только первый вклад, мы можем переписать уравнение (6) в виде

$$
\begin{aligned}
W_{n}(k) & =\frac{2 \pi}{\hbar} U_{0}^{2} a^{3} \\
& \times \sum_{n^{\prime}} \int d z \cdot q(z)[1-q(z)] R_{n n^{\prime}}(z) g_{n^{\prime}}\left(E_{n}(k)\right) .
\end{aligned}
$$

Плотность состояний в $n^{\prime}$-подзоне ГС определяется выражением

$$
g_{n^{\prime}}(E)=\frac{1}{(2 \pi)^{2}} \int d^{2} k^{\prime} \delta\left(E_{n^{\prime}}\left(k^{\prime}\right)-E\right) .
$$

Для ГС при параболической аппроксимации закона дисперсии и положительной эффективной массе носителя $m_{\lambda}$, можно записать

$$
E_{\lambda}(k)=E_{\lambda}^{(0)}+\frac{\hbar^{2} k^{2}}{2 m_{\lambda}}
$$

Тогда для плотности состояний (на единицу площади ГС) получаем

$$
g^{(0)}=\sum_{\lambda} \frac{m_{\lambda}}{2 \pi \hbar^{2}} \vartheta\left[(E)-E_{\lambda}^{(0)}\right] .
$$

Здесь $\vartheta(E)$ - единичная ступенчатая функция Хевисайда, $E_{\lambda}^{(0)}-$ положение края (при $\left.k=0\right) \lambda$-подзоны, а 


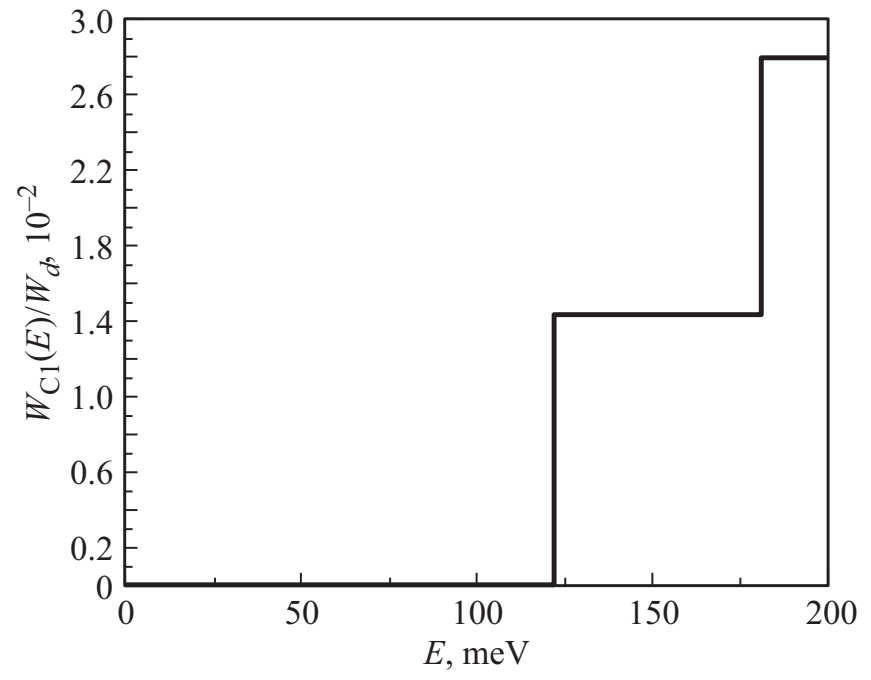

Рис. 1. Зависимость вероятности рассеяния в единицу времени $W_{\mathrm{C} 1}(E)$ для электрона в нижней подзоне размерного квантования $\mathrm{C} 1 \quad$ ГC $\mathrm{Al}_{0.3} \mathrm{Ga}_{0.7} \mathrm{As}-\mathrm{GaAs}$ типа 4-4-4 на глубокой донорной примеси Те от энергии электрона $E$. $W_{d}=\left(U_{0}^{(d)} a\right)^{2} m_{0} q / 4 \hbar^{3}=1.59 \cdot 10^{10} \mathrm{~s}^{-1}$; обе КЯ ГС легированы глубокой примесью Те с постоянным профилем $q$, внутренний и внешние барьерные слои ГС - постоянным профилем $0.1 q$; $q=10^{-5}-$ средняя атомная примесная концентрация, соответствующая объемной концентрации $10^{17} \mathrm{~cm}^{-3}$.

$m_{\lambda}$ - соответствующая эффективная масса. В случае, когда для дырочной подзоны $m_{\lambda}<0$, выражение для закона дисперсии (11) становится неприменимым, и при малых $k$ закон дисперсии в такой подзоне можно аппроксимировать выражением

$$
E_{\lambda}(k)=E_{\lambda}^{(0)}+\frac{\hbar^{2} k^{2}}{2 m_{\lambda}}+\frac{\hbar^{2} b_{\lambda}^{2}}{2 m_{0}} k^{4} .
$$

Типичные значения феноменологического параметра $b_{\lambda}$ могут быть заключены между $10^{-7}$ и $10^{-6} \mathrm{~cm}$ [9]. Вычисление интеграла (10) для плотности состояний (на единицу площади ГС) с законом дисперсии (13) приводит к выражению

$$
\begin{aligned}
g^{(-)}(E)= & \frac{m_{0}\left[E-E_{\lambda}^{(0)}+E_{\lambda, 0}\right]^{-1 / 2}}{4 \pi\left|m_{\lambda}\right| b_{\lambda}^{2} E_{\lambda, 0}^{1 / 2}} \\
& \times \begin{cases}1, & E_{\lambda}^{(0)}-E_{\lambda, 0} \leq E<E_{\lambda, 0}, \\
0.5, & E>E_{\lambda}^{(0)} .\end{cases}
\end{aligned}
$$

Здесь: $E_{\lambda, 0}=\left(m_{0} / 2 m_{\lambda}\right)^{2} \hbar^{2} / 2 m_{0} b_{\lambda}^{2}$.

Профиль легирования ГС определяется 1D-примесной концентрацией $q(z)$, фактор $[1-q(z)]$ в уравнении (9) учитывает корреляцию примесных атомов внутри ячейки, то есть невозможность встраивать более одного примесного атома в одну и туже элементарную ячейку. При условии, что $q \ll 1$ уравнение (9) описывает суммарное некогерентное рассеяние, тогда как при $q \rightarrow 1$ вероятность 2D-электронного рассеяния становится равной нулю, поскольку этот случай соответствует почти идеальной 2D-периодической структуре.

На рис. 1 приведен график зависимости вероятности рассеяния в единицу времени на глубокой донорной примеси для электрона $W_{\mathrm{Cl}}(E)$ в подзоне размерного квантования С1 от энергии носителя $E$ для симметричной полупроводниковой ГС $\mathrm{Al}_{0.3} \mathrm{Ga}_{0.7} \mathrm{As}-\mathrm{GaAs}$ типа 4-4-4 с двумя КЯ (ширина левой и правой КЯ равна $4 a$, внутреннего барьера - $\left.4 a, a=5.655 \cdot 10^{-8} \mathrm{~cm}\right)$, полученный численным решением выражения (9) при $n=\mathrm{C} 1$ и $n^{\prime}=\mathrm{C} 1, \mathrm{C} 2$.

На рис. 2 приведен график зависимости вероятности рассеяния в единицу времени на глубокой акцепторной примеси для дырки $W_{\mathrm{HH} 1}(E)$ в подзоне размерного квантования НН1 от энергии носителя $E$ для полупроводниковой ГС $\mathrm{Al}_{0.3} \mathrm{Ga}_{0.7} \mathrm{As}-\mathrm{GaAs}$ типа 7-2-5 с двумя КЯ на основе численного решения выражения (9) при $n=\mathrm{HH} 1$ и $n^{\prime}=\mathrm{HH} 1, \mathrm{HH} 2, \mathrm{HH} 3, \mathrm{LH} 1, \mathrm{LH} 2$. Предполагается, что положение уровня Ферми и, следовательно, заполнение подзон размерного квантования в обеих ГС определяется, как обычно, уровнем легирования мелкими примесями, расположенными во внешних барьерных слоях и отделенными от системы КЯ спейсерами соответствующей ширины. Расчеты выполнены для случая предельно низких температур $(4.2 \mathrm{~K})$, когда темп примесного рассеяния может существенно превышать темп рассеяния на фононах.

Для нахождения вероятностей примесного рассеяния в единицу времени $W_{\mathrm{C} 1}(E)$ и $W_{\mathrm{HH} 1}(E)$ необходимо было

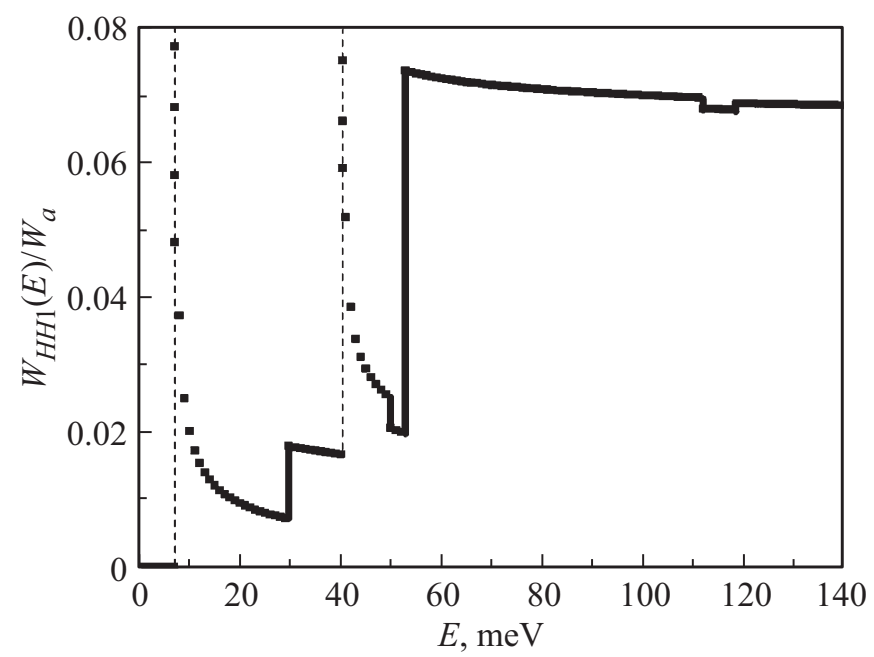

Рис. 2. Зависимость вероятности рассеяния в единицу времени $W_{\mathrm{C} 1}(E)$ для дырки в нижней подзоне размерного квантования HН1 ГC $\mathrm{Al}_{0.3} \mathrm{Ga}_{0.7} \mathrm{As}-\mathrm{GaAs}$ типа 7-2-5 на глубокой акцепторной примеси $\mathrm{Cu}$ от энергии дырки $E$. $W_{a}=\left(U_{0}^{(a)} a\right)^{2} m_{0} q / 7 \hbar^{3}=2.05 \cdot 10^{10} \mathrm{~s}^{-1}$; обе КЯ ГС пролегированы глубокой примесью $\mathrm{Cu}$ с постоянным профилем $q$, внутренний и внешние барьерные слои ГС - постоянным профилем $0.1 q ; q=10^{-5}-$ средняя атомная примесная концентрация, соответствующая объемной концентрации $10^{17} \mathrm{~cm}^{-3}$. Принято: $b_{\mathrm{HH} 2}=b_{\mathrm{LH} 2}=10^{-6} \mathrm{~cm}$. 
Значения энергий $E(\mathrm{meV})$ краев подзон размерного квантования (при $k=0)$ и соответствующие эффективные массы поперечного движения $m_{\lambda}$ (в единицах $m_{0}$ ) для электронов, тяжелых и легких дырок для двух типов $Г$ ГC $\mathrm{Al}_{0.3} \mathrm{Ga}_{0.7}-\mathrm{GaAs}$ 4-4-4 и 7-2-5

\begin{tabular}{|c|c|c|c|c|c|c|c|}
\hline \multicolumn{8}{|c|}{ ГС 4-4-4 } \\
\hline $\mathrm{C} 1$ & $\mathrm{C} 2$ & HH1 & HH2 & HH3 & LH1 & LH2 & \\
\hline $\begin{array}{l}121.81 \\
0.0750\end{array}$ & $\begin{array}{l}180.75 \\
0.0755\end{array}$ & $\begin{array}{c}57.16 \\
0.1364\end{array}$ & $\begin{array}{c}65.23 \\
0.1420\end{array}$ & - & $\begin{array}{c}84.97 \\
0.1654\end{array}$ & $\begin{array}{l}131.10 \\
0.1776\end{array}$ & $\begin{array}{c}E, \mathrm{meV} \\
m_{\lambda} / m_{0}\end{array}$ \\
\hline \multicolumn{8}{|c|}{ ГС 7-2-5 } \\
\hline $\mathrm{C} 1$ & $\mathrm{C} 2$ & HH1 & HH2 & HH3 & LH1 & LH2 & \\
\hline $\begin{array}{c}74.66 \\
0.0705\end{array}$ & $\begin{array}{l}154.23 \\
0.0724\end{array}$ & $\begin{array}{c}29.68 \\
0.1407\end{array}$ & $\begin{array}{c}49.86 \\
-0.1002\end{array}$ & $\begin{array}{l}118.63 \\
0.0207\end{array}$ & $\begin{array}{c}52.85 \\
0.0443\end{array}$ & $\begin{array}{c}111.92 \\
-0.0302\end{array}$ & $\begin{array}{c}E, \mathrm{meV} \\
m_{\lambda} / m_{0}\end{array}$ \\
\hline
\end{tabular}

определить феноменологический параметр $U_{0}$ для глубокой донорной и глубокой акцепторной примеси: $U_{0}^{(d)}$ и $U_{0}^{(a)}$ соответственно. Это сделано простым путем, используя экспериментально определенные значения энергий связи примесных состояний, которые принимались равными $100 \mathrm{meV}$ для глубокой донорной примеси Tе и $150 \mathrm{meV}$ для глубокой акцепторной примеси $\mathrm{Cu}$ в квантовой яме GaAs [10,11].

В таблице приведены значения энергий краев подзон размерного квантования для электронов, тяжелых и легких дырок ГC $\mathrm{Al}_{0.3} \mathrm{Ga}_{0.7} \mathrm{As}-\mathrm{GaAs}$ типов 4-4-4 и 7-2-5, отсчитанные от дна соответствующей КЯ и измеренные в $\mathrm{meV}$, а также значения эффективных масс в единицах массы свободного электрона $m_{0}$. Эффективные массы дырок вычислялись с использованием известного гамильтониана Латтинджера [12] в модели $(4 \times 4)$ [13], недиагональные по спинорным индексам элементы которого приводили к смешиванию состояний тяжелых и легких дырок ГС, вклад которых рассчитывался по теории возмущений до второго порядка включительно.

Зависимость вероятности рассеяния в единицу времени на глубокой примеси для электронов от энергии носителя повторила кусочно-постоянный вид плотности состояний подзон С1 и С2 размерного квантования ГС 4-4-4 с учетом вклада интеграла перекрытия волновых функций (7) при заданном профиле легирования $q(z)$ ГС. Для ГС 7-2-5 дырочные подзоны HН2 и LH2 имеют отрицательные эффективные массы, а вероятности рассеяния в единицу времени на глубокой акцепторной примеси для дырок у краев этих подзон имеют сингулярности, характерные для одномерных систем; дырочные подзоны HH1, HH3, LH1 имеют положительные эффективные массы, вероятность рассеяния в единицу времени здесь снова повторяет кусочно-постоянный вид плотности состояний этих подзон.

\section{3. Заключение}

Оптические и кинетические свойства электронов и дырок в системах КЯ полупроводниковой ГС сильно зависят от параметров глубоких уровней, например, через изменение уровня Ферми и изменение характера рассеяния носителей на примесях [14]. Кроме того, сама зонная структура полупроводника и симметрия глубокой примеси в явном виде определяют тип квантового состояния примеси, причем для полного описания состояния примеси нужно учитывать неблоховские состояния с энергиями в запрещенной зоне, которые соответствуют примесному ядру [15]. Недавние исследования термостимулированной проводимости ГC InGaAs-GaAs [2] показали, что глубокие ловушки могут играть заметную роль в процессе рекомбинации и механизме фотопроводимости. Формализм тунннельного гамильтониана использован в [16] для расчета скоростей фотопроводимости в ГС с глубокими КЯ с зонной диаграммой типа I между состояниями в валентной зоне в КЯ и уровнями глубоких примесных центров. Показано, что скорость фотозарядки примесей резко возрастает, когда частота света превышает определенный порог. Исследование формирования резонансных состояний в зоне проводимости, индуцированных глубокими примесями вне КЯ ГС в [17] показало, что коэффициенты захвата и рассеяния могут изменяться на несколько порядков в узких энергетических интервалах, создавая ярко выраженный и сильный механизм рассеяния.

Отметим, что проведенное в настоящей работе исследование рассеяния электронов и дырок в прямозонных полупроводниковых системах КЯ на глубоких примесях в приближении короткодействующего потенциала примеси и метода эффективной массы, проводилось без каких-либо ограничений по профилю легирования ГС.

\section{Конфликт интересов}

Автор заявляет, что у него нет конфликта интересов.

\section{Список литературы}

[1] Y. Zhang. Chin. Phys. B 27, 117103-1 (2018).

[2] S.A. Iliash, S.V. Kondratenko, A.S. Yakovliev, Vas.P. Kunets, Yu.I. Mazur, G.J. Salamo. Semicond. Phys. Quantum Electron. Optoelectron. 19, 75 (2016).

[3] Y. Zhang, J. Wang. Phys. Rev. B 90, 155201 (2014). 
[4] P.M. Koenrad, M.E. Flatté. Nature Mater. 10, 91 (2011).

[5] Ł. Gelczuk, M. Dabrowska-Szata, B. Sciana, D. Pucicki, D. Radziewicz, K. Kopalko, M. Tłaczała. Mater. Sci. Poland. 34, 726 (2016).

[6] N. Miyashita, Y. He, N. Ahsan, Y. Okada. J. Appl. Phys. 126, Art. № 143104 (2019).

[7] Л.В. Келдыш. ЖЭТФ 45, 364 (1964).

[8] И.М. Лифшиц. ЖЭТФ 17, 1017 (1947).

[9] V.I. Belyavsky, Yu.V. Kopaev, N.V. Kornyakov, Yu.A. Pomerantsev, S.V. Shevtsov. Semicond. Sci. Technol. 13, 460 (1998).

[10] В.И. Белявский, Ю.В. Копаев, Н.В. Корняков, С.В. Шевцов. Письма в ЖЭТФ 61, 1004 (1995).

[11] В.И. Белявский, Ю.В. Копаев, Ю.А. Померанцев, С.В. Шевцов. Письма в ЖЭТФ 62, 197 (1995).

[12] J.M. Luttinger. Phys. Rev. 102, 1030 (1956).

[13] L.C. Andreani, A. Pasquarello, F. Bassani. Phys. Rev. B 36, 5887 (1987).

[14] G. Bastard, J.A. Brum, R. Ferreira. Solid State Phys. 44, 229 (1991).

[15] J.C. Inkson. J. Phys. C 13, 369 (2000).

[16] E.Y. Perlin, A.A. Popov. J. Opt. Technol. 81, 365 (2014).

[17] A. Blom, M.A. Odnoblyudov, I.N. Yassievich, K.A. Chao. Phys. Rev. B Condens. Matter. 65, 155302 (2002).

Редактор Т.Н. Василевская 